\title{
Synthesis of Fuzzy Controller for Cross-Flow Water Turbine
}

\section{K.Ormandzhiev, S. Yordanov, S. Stoyanov}

Key Words: Automatic system; cross-flow turbine; transient processes; fuzzy controller.

Abstract. The paper deals with a developed mathematical model describing the operation of automatic system for controlling of cross-flow water turbine in laboratory conditions. Fuzzy governor is synthesized and the transient processes in the system are compared toward these ones during utilization of classical PD controller. The results from numerical experiment are presented in graphical form.

\section{Introduction}

The automatic electro-hydraulic systems with throttle governing find wide application in the systems for controlling of the revolutions of water turbines from various types. Their executive devices are usually hydraulic motors (hydro cylinders) with reciprocating rectilinear motion. The flexible control and the opportunity for easy formation of the necessary law of governing which are achieved by the electronic devices are combined with large output powers and good dynamic characteristics of the hydraulic mechanisms and systems.

The problems for governing of water turbines and connected pressure equipments are an object of study by many authors $[6,8,9,10,16]$. The non-stationary processes originating in hydro-power plant exert considerable influence on the operation of water turbines, especially in the cases, when they are fed by common pressure pipeline
$[6,8,11,13]$. Performance of various centrifugal pumps, which can be used in the study of pressure systems to supply water turbines under laboratory conditions, were obtained in $[7,14]$. The conditions for operation of automatic systems of water turbines in transient modes are quite unfavourable. In the first place this is due to the originating hydraulic shock in the pressure pipelines and to the large variety of operation modes of turbines. At these conditions the achievement of definite quality of transient processes regarding the change of revolutions can be at the account of considerable increase of the head, which can reach quite large values in some cases. This imposes a synthesis of optimum law of controlling to be performed $[11,12,13]$ or parametric tuning of automatic governors of water turbines at introduction of optimality criterion. As a result of the system optimization the best quality of transient processes is achieved both over the change of revolutions and over the head in supply pipelines. In [3] is presented an algorithm based on Kalman method, which significantly improves the dynamic accuracy of the measured signals in the system.

The fuzzy logical control is outlined as one of the most active and purposive studying directions in the theory of fuzzy sets. The fuzzy governors are applied successfully during control of series of technological objects and processes. That is why, the studying of fuzzy control and its comparison with the conventional control as well the type of continuous law of governing are of essential significance about the up-to-date theory of controlling $[1,2,4,5,15]$.

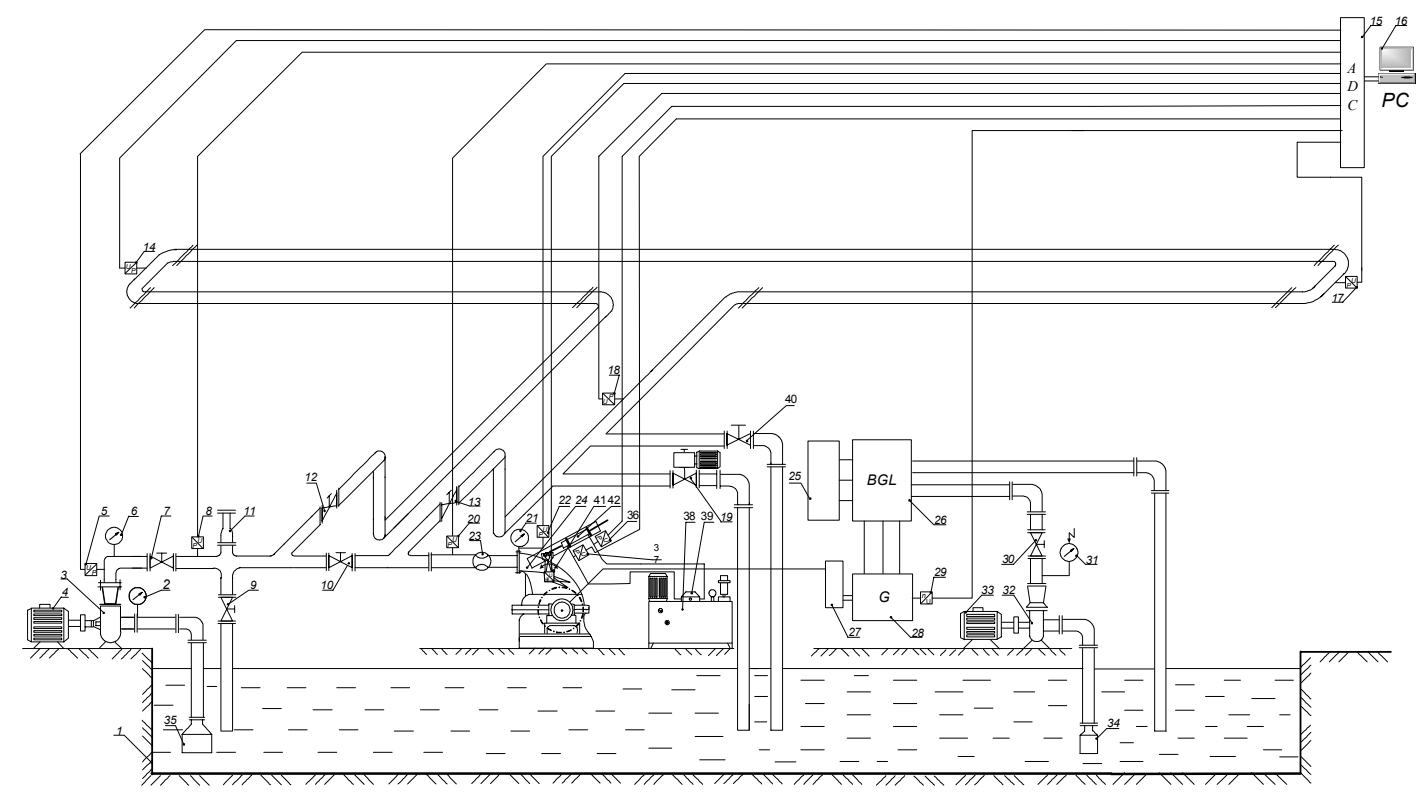

Figure 1. Scheme of the automatic system 


\section{Physical Implementation Mathematical Model}

The scheme of laboratory stand of the automatic system for governing of the revolutions of cross-flow water turbine is presented in figure 1. For controlling of the system PD governor or FUZZY governor can be used.

The system consists of the following elements: 1 - reservoir; 2 - vacuum meter; 3 - centrifugal pump; 4 - electric motor; 5, 8, 14, 17, 18, 20, 22 - transducers for low pressure; 6, 21 - manometers; 7, 9, 10, 12, 13, 30, 40 valve door; 15 - analogue-to-digital converter (ADC); 16 - computer (PC); 19 - valve door with electric drive; 23 - flowmeter; 24 - cross-flow water turbine; 25 - electric panel; 26 - generator load block (GLB); 27 - belt gearing; 28 - three-phase synchronous generator; 29 - transducer for rotation frequency of the generator shaft; 31 - contact manometer; 32 - centrifugal pump for cooling of the block for loading of generator; 33 - electric motor; 34, 35 - inlet flaps; 36, 37 - transducers for high pressure; 38 - hydrostation; 39 - servovalve; 41 - transducer for rotation of the governing blade at the input of turbine; 42 - hydraulic cylinder (servomotor).

Depending on the position of valve doors 10, 12 and 13 schemes with short and long pressure pipeline are realized. A scheme with short pressure pipeline is accomplished at the open position of valve door 10 and closed position of valve door 12 and 13. The supplied flow from the basic centrifugal pump 3 passes towards the turbine 17 through short pressure pipeline with diameter $d=200 \mathrm{~mm}$ and length $l=5.2 \mathrm{~m}$. A scheme with long pressure pipeline is accomplished at closed position of valve door 10 and open position of valve door 12 and 13. The sector of pressure pipeline between valve doors 12 and 13 is made by PVC with diameter $d=100 \mathrm{~mm}$ and length $l=48 \mathrm{~m}$. Disturbances in the system can be introduced by means of a change of the loading moment from the block for loading of generator as well by means of a change of the flow entering towards the water turbine - valve door 19 and 40 (figure 1). The scheme of automatic governor of turbine is shown in figure 2. It consists of the following elements: 1 - gear pump; 2 safety valve; 3 - manometer; 4 - filter for high pressure; 5 - servovalve; 6 - servomotor; 7 - filter for low pressure; 8 - reservoir; 9 - cross flow water turbine; SD -setting device; AD - adding device; EG - electronic governor; $\mathrm{TO}$ - transducer for rotation frequency of the turbine shaft; TR - transducer for rotation of the governing blade at the turbine input.

The operation of governor (figure 2) is as follows: In steady-state mode, on a definite value of the setting voltage $u_{3}$ corresponds a determinate angular velocity of rotation of the turbine shaft $\omega_{0}$. Transient processes in the system will occur during a change of: the loading moment which is applied towards the turbine shaft; the hydraulic parameters (head $h_{\mathrm{T}}$ or flow $q_{\mathrm{T}}$ ) at the turbine input; the setting signal - the setting voltage $u_{3}$.

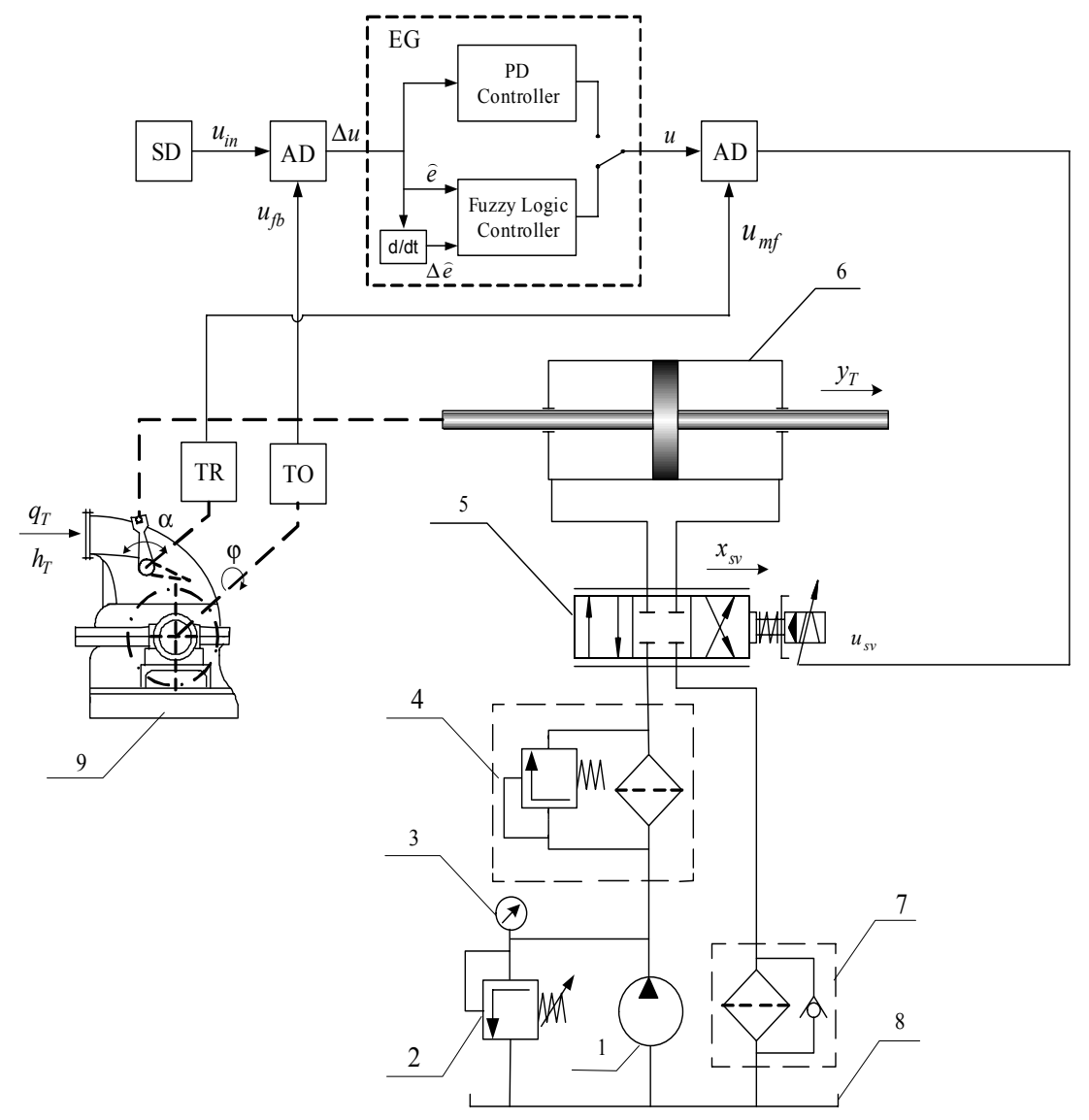

Figure 2. Scheme of the automatic governor 


\section{Pressure System with Concentrated Parameters and Availability of Two Branchings}

For realizing of automatic system with short pressure pipeline (figure 1) it is necessary valve door 10 to be open, but valve doors 12 and 13 to be closed. The equivalent hydraulic scheme is shown in figure 3.

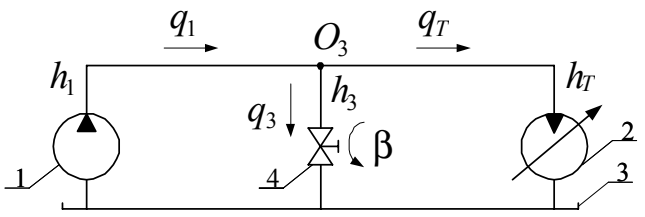

Figure 3. Scheme of pressure system with short pressure pipeline and two branchings 1 - turbopump; 2 - cross-flow water turbine; 3 - reservoir; 4 - valve door

Since the length of pipeline from the turbopump to the common point of branchings is much larger than the lengths of the same branchings, then for this case a combined model with distributed and concentrated parameters is developed. Irrespective of the type of scheme of the pressure system (figure 1), the operation of turbopump 3 and water turbine 24 have to be accounted for as boundary conditions at the beginning and at the end of pressure pipeline.

- Equation for the connection between the hydraulic parameters of turbopump. The characteristic of turbopump at constant rotation frequency of its shaft can be described with full parabola from the following type:

(1) $h_{1}=A_{1}-A_{2} q_{1}-A_{3} q_{1}^{2}$

where $h_{1}, q_{1}$ - non-dimensional head and non-dimensional flow of the turbopump; $A_{1}, A_{2}, A_{3}$ - coefficients.

- Equation for the passing flow through the turbine

(2) $q_{T}=\frac{\lambda y_{T}-y_{T}^{2}}{\lambda-1} \sqrt{h_{T}}$

where $h_{T}, q_{T}$ - non-dimensional head and non-dimensional flow at the input of turbine; $y_{T}$ - relative rotation of the governing blade at the input of turbine; $\lambda$-constructive coefficients.

- Equation for the non-stationary motion in the pressure pipeline from the pump up to the common point $\left(\mathrm{p} . \mathrm{O}_{3}\right)$ of branchings

(3) $T_{L 1} \frac{d q_{1}}{d t}+k_{f 1} q_{1}^{2}=h_{1}-h_{3}$

where $h_{3}$ - non-dimensional head in the common point of branchings; $T_{L 1}$ - time constant of the pipeline; $k_{f 1}$ - coefficient of friction.

- Equation for the non-stationary motion in the branching toward the turbine (from $\mathrm{p} . \mathrm{O}_{3}$ up to the turbine):

(4) $T_{L 2} \frac{d q_{T}}{d t}+k_{f 2} q_{T}^{2}=h_{3}-h_{T}$

where $T_{L 2}$ - time constant of the pipeline; $k_{f 2}$ - coefficient of friction.

- Equation for the passing flow through valve door 4 of the branching.
Since the length of branching is too small then for the passing flow through it in dimensional type can be written as follows:

$$
\text { (5) } Q_{3}=\mu_{3} f_{3}\left(\beta_{3}\right) \sqrt{2 g H_{3}}
$$

where $\mathrm{Q}_{3}$ - flow through the branching; $\mu_{3}$ - coefficient of the flow of valve door; $\beta_{3}$ - angle of rotation of the valve door; $f_{3}\left(\beta_{3}\right)$ - area of the light section of valve door, $f_{3}=k_{\beta} \beta_{3} ; k_{\beta}$ - coefficient of proportionality; $H_{3}$ - head at the input of valve door. After transformation for the passing flow in non-dimensional type is obtained

(6) $q_{3}=\beta \sqrt{h_{3}}$

where $q_{3}=\frac{Q_{3}}{Q_{30}} ; h_{3}=\frac{H_{3}}{H_{30}} ; \beta=\frac{\beta_{3}}{\beta_{3 \max }}$

- Equation for continuity (in p. $\mathrm{O}_{3}$ )

(7) $q_{1}=q_{T}+q_{3}$.

\section{Control System}

The mathematical model of automatic governor of turbine (figure 2) is described by means of the following equations in non-dimensional type:

- Equation of the adding device:

(8) $\Delta u=u_{i n}-u_{f b}$

where $\Delta u$ - voltage at the output of adding device; $u_{f b}-$ voltage of the feedback, $u_{f b}=k_{f b} y_{T} ; k_{f b}$ - amplification coefficient of the global feedback.

- Equation of the electronic PD controller

(9) $u=k\left(\Delta u+T_{d} \frac{d \Delta u}{d t}\right)$

where $k, T_{d}$ - coefficient of amplification and time constant of differentiation of the governor; $u$-voltage at the output of governor.

- Equation of the servovalve

(10) $T_{s v} \frac{d x_{s v}}{d t}+x_{s v}=k_{s v} u_{s v}$

where $u_{s v}$ - non-dimensional input voltage of the servovalve; $T_{s v}$ - time constant of the servovalve; $k_{s v}-$ coefficient of amplification.

- Equation of the servomotor

$$
T_{T}^{2} \frac{d^{2} y_{T}}{d t^{2}}+2 \zeta_{T} T_{T} \frac{d y_{T}}{d t}+y_{T}=\frac{1}{T_{S}} \int_{0}^{t} x d t
$$

where $T_{S}$ - time constant of the servomotor; $T_{T}, \xi_{T}$ - time constant and coefficient of damping of the load.

- Equation of the adding device of the local feedback:

(12) $u_{s v}=u-u_{m f}$

where $u_{m f}$ - voltage of the local feedback, $u_{m f}=k_{m f} y_{T} ; k_{m f}$ - coefficient of amplification.

\section{Object of Governing}

- Equation for rotation of the turbine shaft

(13) $T_{A} \frac{d \varphi}{d t}=\frac{q_{T} h_{T}}{\varphi}-m_{G}-k_{C}-k_{M} \varphi^{2}$ 

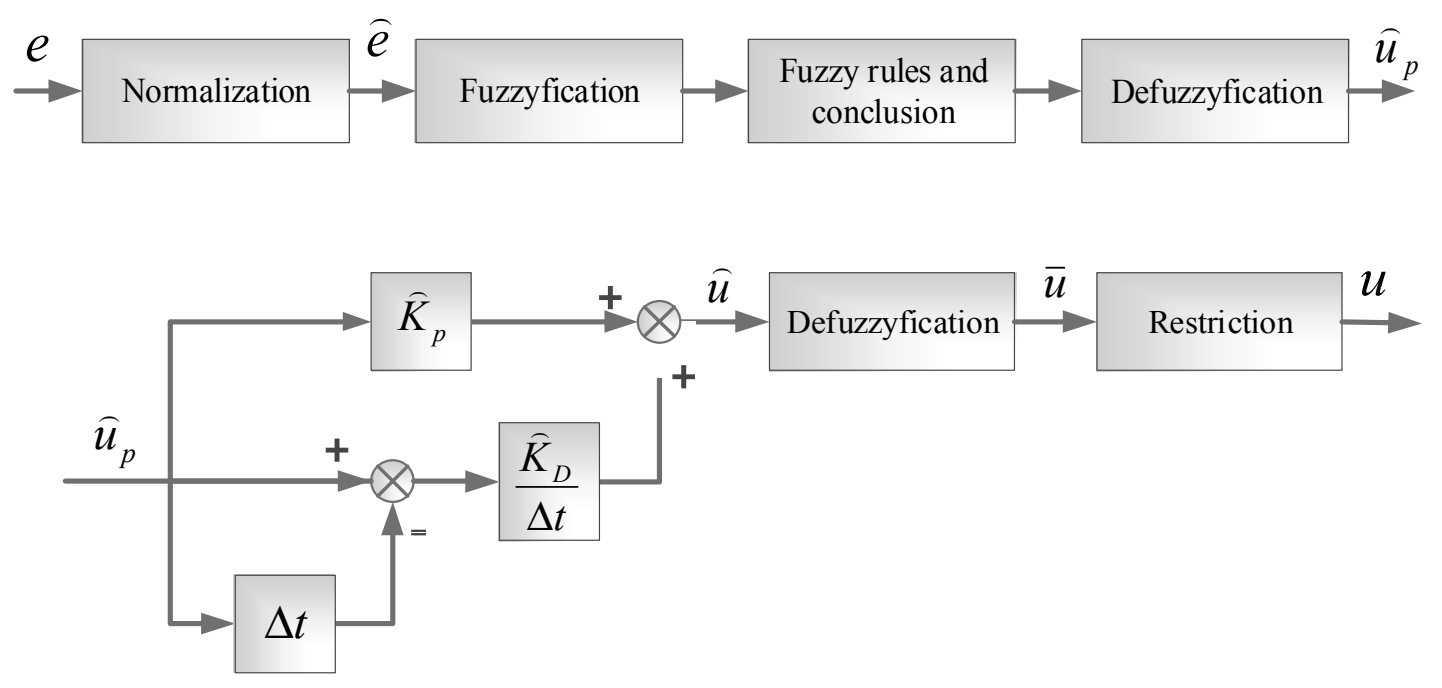

Figure 4. Structure of a fuzzy PD controller

where $\varphi, m_{G}-$ non-dimensional frequency of the turbine shaft; non-dimensional loading moment of the generator; $T_{A}$ - time constants of the aggregate; $k_{C}, k_{M}$ - coefficients.

The joint solution of equations from the mathematical model affords an opportunity for theoretical determination of the transient processes flowing in the automatic system at different configurations of the pressure system.

\section{System for Control}

At classical controlling of water turbine, usually the governing is based on the revolutions of generator shaft $\varphi$ as the global feedback and the angle of blade rotation $\varphi$ as well as local feedback having a stabilizing role. In the developed governors, two quantities also enter, i.e. the head on output of the water pump $h_{1}$ and the head on input of the water turbine $h_{T}$. Thus, the designed governor can also be used at the system: pressure tower (dam lake) - pressure penstock - water turbine. At this system $h 1$ will be the head on the input of derivation (intake system).

The purpose of this work is the synthesis of a fuzzy controller for management of the object.

\section{Design of a Fuzzy PD Controller}

The goal in designing a fuzzy controller is to achieve the desired management by means of the regulator with the most simple structure and at least count of tuning parameters. Figure 4 shows the structure of fuzzy PD controller with one input and one output variables.

Input variable for the fuzzy controller is the error $e(n)=u_{i n}(n)-u_{f b}(n)$, (is the preset action, $\mathrm{y}(\mathrm{n})$ is the output variable of the system) and the output variable of the regulator is $u(n)$. The normalized control action shall be expressed with the dependence

$$
\widehat{u}(n)=\widehat{K}_{p} \widehat{u}_{p}(n)+\widehat{K}_{D} \frac{\Delta \widehat{u}_{p}(n)}{\Delta t}, n=0,1,2 \ldots
$$

where $\hat{K}_{P}$ and $\hat{K}_{D}$ are the normalized coefficients for the proportional and differential components. Normalized error is defined as

$$
\hat{e}(n)=\left\{\begin{array}{cc}
1, & s_{e} e(n)>1 \\
s_{e} e(n), & \left|s_{e} e(n)\right| \leq 1 \\
-1, & s_{e} e(n)<-1
\end{array}\right.
$$

where $s_{e}=|r(0)-y(0)|, r(0)$ and $y(0)$ are the initial values of the preset action and of the manageable value.

The denormalization of the control action $\hat{u}(n)$ on the dependence $\bar{u}(n)=s_{u} \widehat{u}(n)$, where $s_{u}$ is the coefficient of denormalization.

Fuzzy PD controller calculates the control signal based on two inputs - the error of the closed system and its derivative. In this particular case, the error is associated with the deviation of the generator shaft turning angular velocity from the set angular velocity, i.e. this is the error between the angular velocity and the actual angular velocity of rotation of the generator shaft. Then the error derivative is the angular acceleration of the generator shaft. The logic of the fuzzy PD controller is explained by a table composed of nine fuzzy rules (table 1) [2]. Rules may also

Table 1. Table with rules, building the logic of fuzzy PD

\begin{tabular}{|c|c|c|c|}
\hline$\Delta \hat{e}^{\hat{e}}{ }^{\hat{e}}$ & Positive & Zero & Negative \\
\hline Positive & $\begin{array}{l}\text { No } \\
\text { control } \\
\text { signal }\end{array}$ & $\begin{array}{c}\text { Negative } \\
\text { control } \\
\text { signal }\end{array}$ & $\begin{array}{c}\text { Large } \\
\text { negative } \\
\text { control } \\
\text { signal }\end{array}$ \\
\hline Zero & $\begin{array}{c}\text { Positive } \\
\text { control } \\
\text { signal }\end{array}$ & \begin{tabular}{|c|} 
No \\
control \\
signal
\end{tabular} & $\begin{array}{c}\text { Negative } \\
\text { control } \\
\text { signal }\end{array}$ \\
\hline Negative & $\begin{array}{c}\text { Large } \\
\text { positive } \\
\text { control } \\
\text { signal }\end{array}$ & $\begin{array}{c}\text { Positive } \\
\text { control } \\
\text { signal }\end{array}$ & $\begin{array}{c}\text { No control } \\
\text { signal }\end{array}$ \\
\hline
\end{tabular}
controller 
be more (for example, if you need a more precise choice of controller ratios, or range of rules). Controlling rules are intuitive. The large set angle (deviation angle) and the angular velocity of rotation of the turbine shaft (angular velocity) are equal to zero and therefore it is not necessary to provide control signal. There is deviation of the angle in the upper left and the lower right corner, but the angular velocity shows that even without control signal, the deviation from the angle rotation decreases. In this case it's better to wait for the system to go to the desired position (without deviation from the rotation angle).

If a control signal is submitted, the desired value will be exceeded. Below the main diagonal, control signal is submitted because either the deviation of the rotation angle tends to occur, or the system has stabilized in a non-zero position. In the lower left corner, the position is unfavorable, if there is no control signal, the angular velocity indicates that the system will gain even greater deviation from the rotation angle. Therefore, to establish the desired position it is necessary to submit a large positive control signal. The same reasoning applies for the cases above main diagonal, but with opposing signs.

The rules for controlling the fuzzy PD controller (in accordance with table 1) are described in the sequence shown below.

For the control action is recorded

$$
u(n)=\left\{\begin{array}{cc}
u_{\max }(n), & \bar{u}(n)>u_{\max } \\
\bar{u}(n), & u_{\min } \leq \bar{u}(n) \leq u_{\max } \\
u_{\min }(n), & \bar{u}(n)<u_{\min }
\end{array}\right.
$$

where $u_{\min }$ and $u_{\max }$ are the possible minimum and maximum values of the input variable of the object for control. The fuzzy regulator concerned has three rules:

$$
\begin{aligned}
& P 1: \text { If }(\hat{e} \text { is } N V) \text { and }(\Delta \hat{e} \text { is } N V) \text { then }\left(\hat{u}_{p} \text { is } Z V\right) \\
& P 2: \text { If }(\hat{e} \text { is } N V) \text { and }(\Delta \hat{e} \text { is } Z V) \text { then }\left(\hat{u}_{p} \text { is } N V\right) \\
& P 3: \text { If }(\hat{e} \text { is } N V) \text { and }(\Delta \hat{e} \text { is } P V) \text { then }\left(\hat{u}_{p} \text { is } N V\right) \\
& P 4: \text { If }(\hat{e} \text { is } Z R) \text { and }(\Delta \hat{e} \text { is } N V) \text { then }\left(\hat{u}_{p} \text { is } P V\right) \\
& P 5: \text { If }(\hat{e} \text { is } Z R) \text { and }(\Delta \hat{e} \text { is } Z V) \text { then }\left(\hat{u}_{p} \text { is } Z V\right) \\
& P 6: \text { If }(\hat{e} \text { is } Z R) \text { and }(\Delta \hat{e} \text { is } P V) \text { then }\left(\hat{u}_{p} \text { is } N V\right) \\
& P 7: \text { If }(\hat{e} \text { is } P V) \text { and }(\Delta \hat{e} \text { is } N V) \text { then }\left(\hat{u}_{p} \text { is } P V\right) \\
& P 8: \text { If }(\hat{e} \text { is } P V) \text { and }(\Delta \hat{e} \text { is } Z V) \text { then }\left(\hat{u}_{p} \text { is } P V\right) \\
& P 9: \text { If }(\hat{e} \text { is } P V) \text { and }(\Delta \hat{e} \text { is } P V) \text { then }\left(\hat{u}_{p} \text { is } Z V\right)
\end{aligned}
$$

and the fuzzy variable " $N V$ " is for "negatively large", " $P V$ ", - for "positively large" and " $Z V$ " - for "approximately zero" values. The membership functions of the variables $\widehat{e}$ and $\hat{u}_{p}$ have a triangular shape, and for $\hat{e}$ the parameters are fixed, and for $\hat{u}_{p}$ are optimal values are sought, figure 5. Hence the fuzzy regulator has only three rules and six parameters for settings as

$$
u(n)=\left\{\begin{array}{c}
0<\widehat{K}_{p} \leq 1, \quad 0<\widehat{K}_{D} \leq 1, \\
0<s_{u} \leq \max \left(\left|u_{\min }\right|,\left|u_{\max }\right|\right), \\
0<\widehat{u}_{1} \leq 1, \quad 0<\widehat{u}_{2} \leq 1 .
\end{array}\right.
$$

The FIS Editor of Fuzzy Logic Toolbox can be used for the implementation of the fuzzy regulator implementation of the fuzzy regulator. There the parameters of the membership functions are introduced to their specific numerical values. The fuzzy regulator is modeled in a Matlab/Simulink environment. The signal $\hat{e}$ is delived in the input, and on the output of the regulator $-\hat{u}_{p}$. The parameters of the triangular membership functions FSE1, FSE2 and FSE3 of input variable are $[-2,-1.0],[-1,0,1]$ and $[0,1,2]$ (left, peak and right value of the triangular functions for appliance), respectively, for NV, ZV and PV. The parameters of the membersip functions of the output variable are $\left[-2 \mathrm{u}_{2},-1,-\mathrm{u}_{2}\right],\left[-\mathrm{u}_{1}, 0, \mathrm{u} 1\right]$ and $\left[\mathrm{u}_{2}, 1,2 \mathrm{u}_{2}\right]$ respectively for $\mathrm{NV}$, $\mathrm{ZV}$ and $\mathrm{PV}$, as the specific values of $\mathrm{u} 1$ and $\mathrm{u} 2$ are determined by an optimization procedure. The sum of the outputs of the membership functions are summarized and defuzzyfication is performed using the method of the center of gravity.
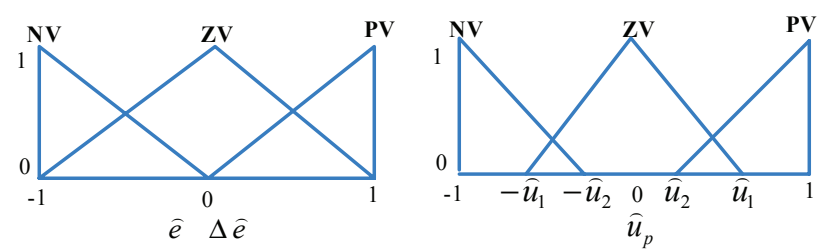

Figure 5. Membership functions of the variables $\widehat{e}$ and $\widehat{u}_{p}$

The synthesized regulator is inserted in the model generated by Matlab SimMechanics.

\section{Setting of Fuzzy PD Regulator}

The parameterization of the fuzzy PD regulator is reduced to solving of the optimization task for satisfaction of given criterion $\mathrm{J}_{\mathrm{T}}$, through the application of the weighting method.

$$
J_{T}=\sum_{k=0}^{n} \omega_{k} J_{k}
$$

Thus the task is reduced to an optimization by one criterion. The use of criteria based only on the error signal in the system hardly could satisfy the conflicting requirements between the accuracy in steady-state and the dynamic performance of the system - time and overregulation. This requires the use of a generalized criterion of type [5]

$$
J_{T}=\omega_{1} \frac{\int_{0}^{T} e^{2}(t) d t}{\max (e(n))}+\omega_{2} \sigma+\omega_{3} \frac{t_{p}}{T}
$$

where $t_{p}$ is the duration of the transition process, s; $\sigma-$ over-regulation, $\% ; T$ - the time for simulation, $\mathrm{s}$.

In a single step-entrance effect is assumed that $\max (e(n))=1$.

The optimization task to setup the fuzzy PD regulator has been solved in Matlab Simulink, where a model of the closed system for automatic regulation has been created. 
The variables $\hat{u}_{1}, \hat{u}_{2}$ are introduced for the membership functions of the output variable of the fuzzy regulator and the variables $\widehat{K}_{p}$ and $\widehat{K}_{D}$, determining the normalized proportional and differential components.

\section{Experimental Studies}

The experiments have been carried out by means of the utilization of preliminarily built block-scheme in the medium of Matlab Simulink which is presented in figure 6 .

The Simulink model of the object for controlling is given in figure 7.

The fuzzy PD governor is realized by means of the block Fuzzy Logic Controller, from the standard library Fuzzy Logic Toolbox of Matlab Simulink. The purpose of control- ling signal is the system to reach to the specification possibly the most quickly and qualitatively - with minimum overcontrol and fluctuations. The results from conducted experiment are shown in figure 8 and figure 9, respectively the reaction of system during change of specification and during change of loading. The values of some more important parameters, at which the numerical experiments are performed, are shown in table 2.

Table 2

\begin{tabular}{|l|l|l|l|l|l|}
\hline$k$ & 1.00 & $T_{s}, s$ & 0.50 & $T_{L 2}, s$ & 0.12 \\
\hline$k_{M}$ & 0.30 & $T_{s v}, s$ & 0.01 & $k_{f 1}$ & 0.010 \\
\hline$T_{d}, s$ & 0.50 & $k_{f b}$ & 2.50 & $k_{f 2}$ & 0.010 \\
\hline$T_{A}, s$ & 2.00 & $k_{m f}$ & 0.10 & $\lambda$ & 4.00 \\
\hline
\end{tabular}

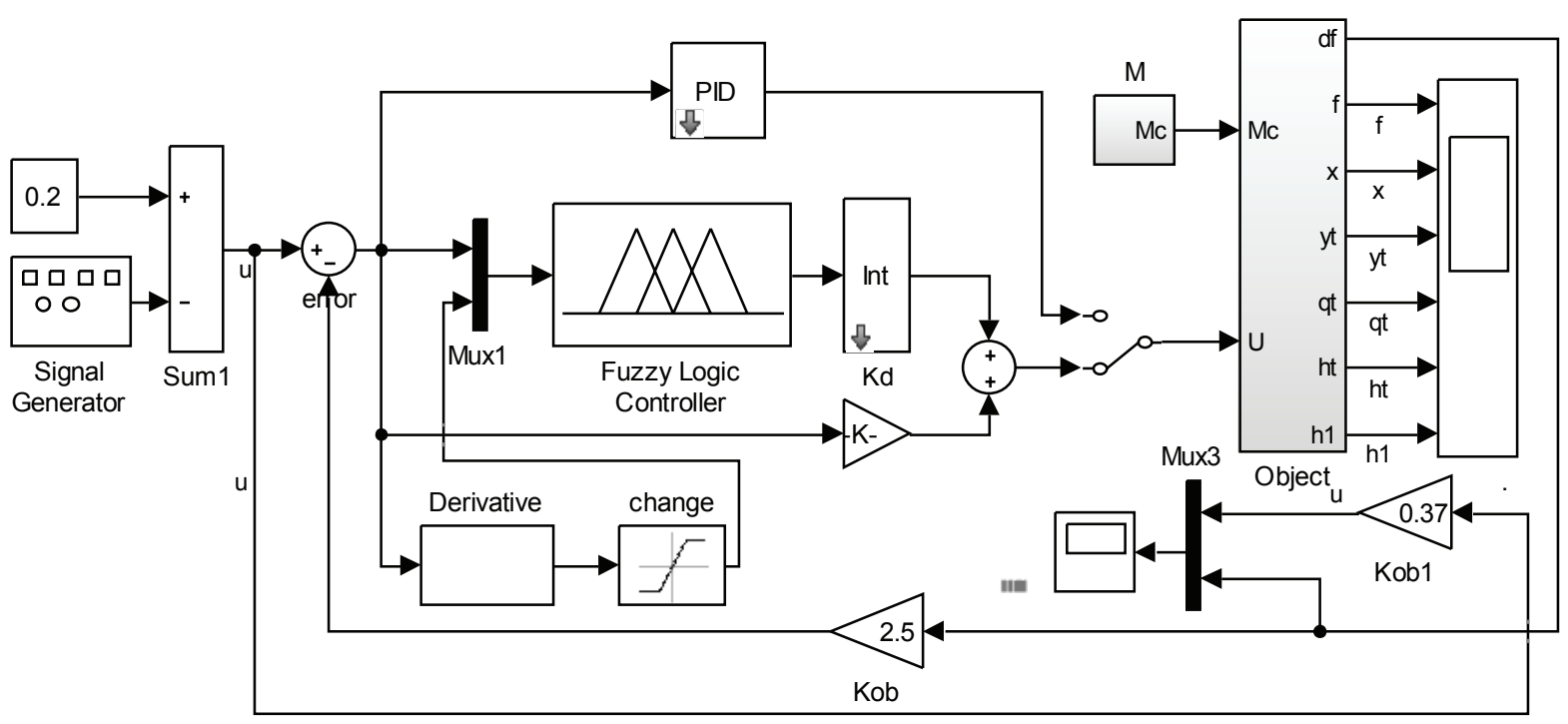

Figure 6. Block-scheme of the servo-system and the fuzzy PD controller

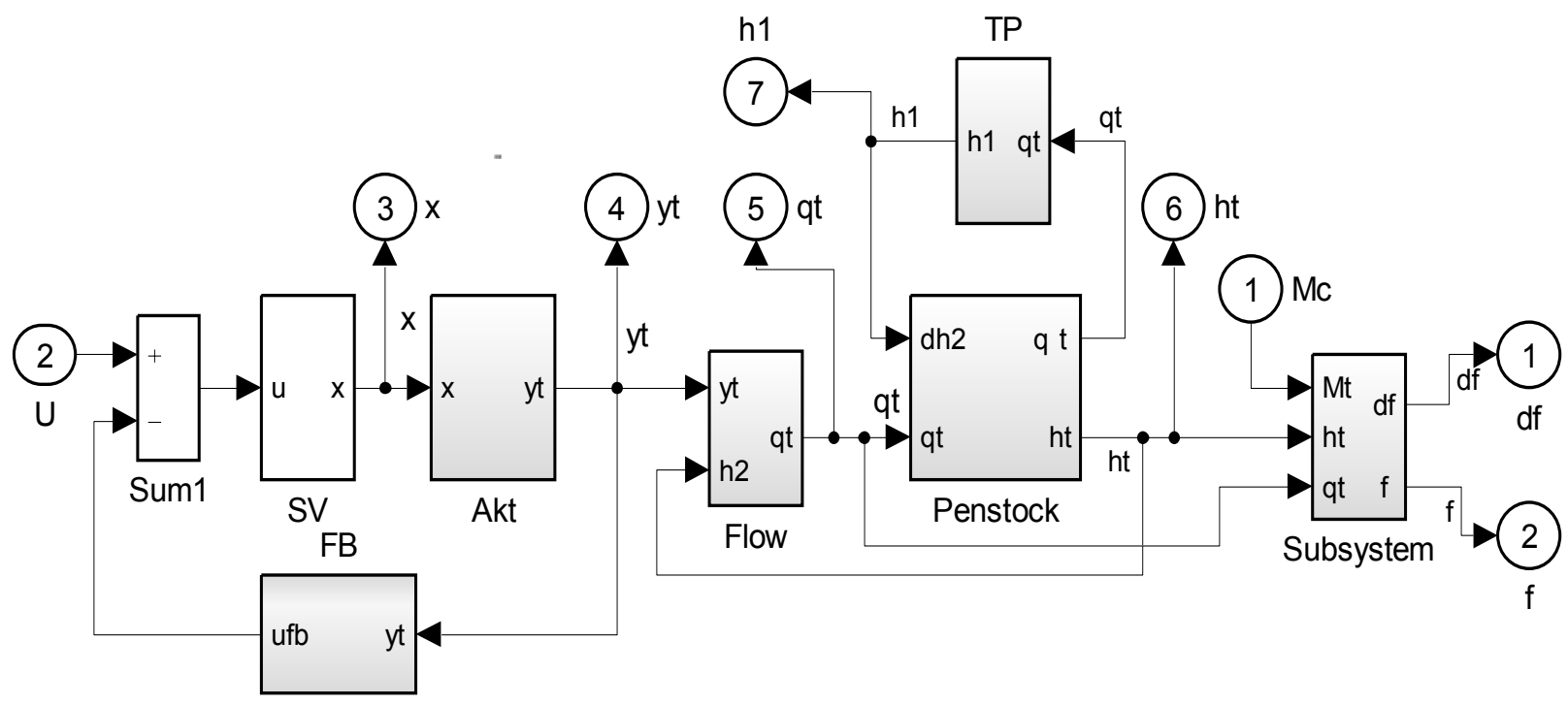

Figure 7. Simulink model of the object 


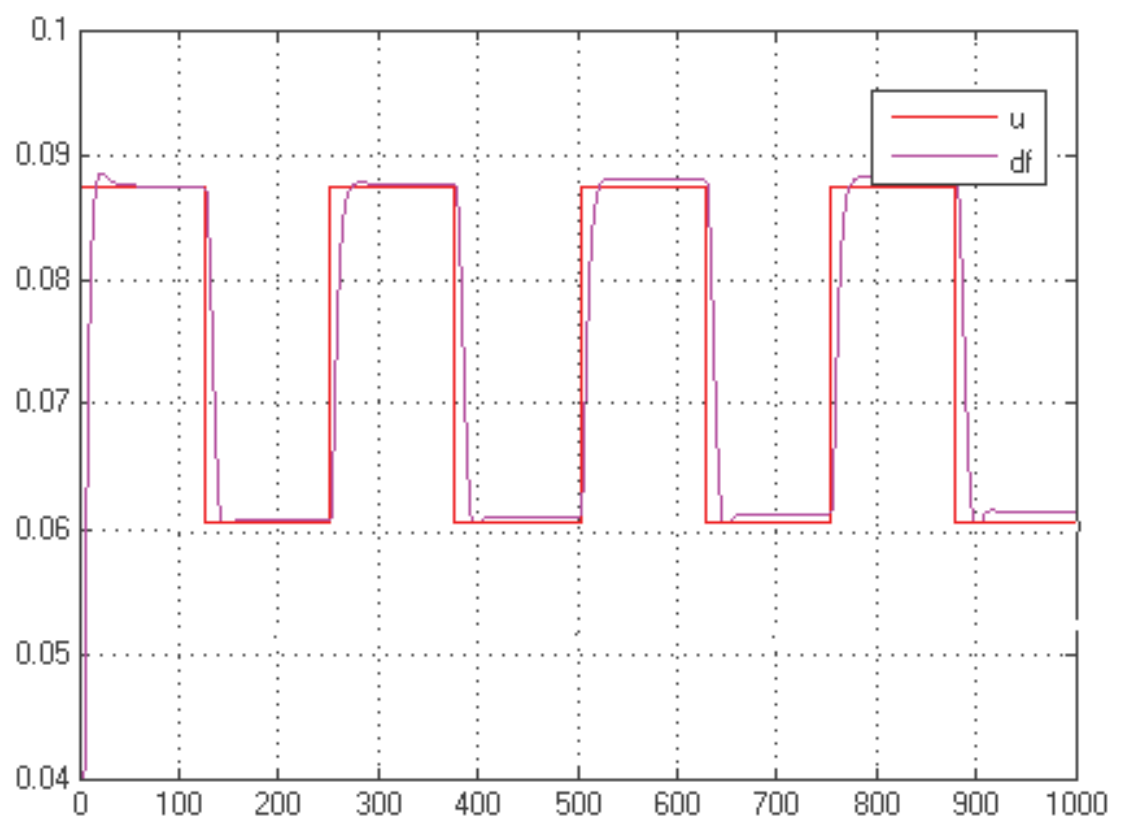

Figure 8. Reaction of system during change of specification

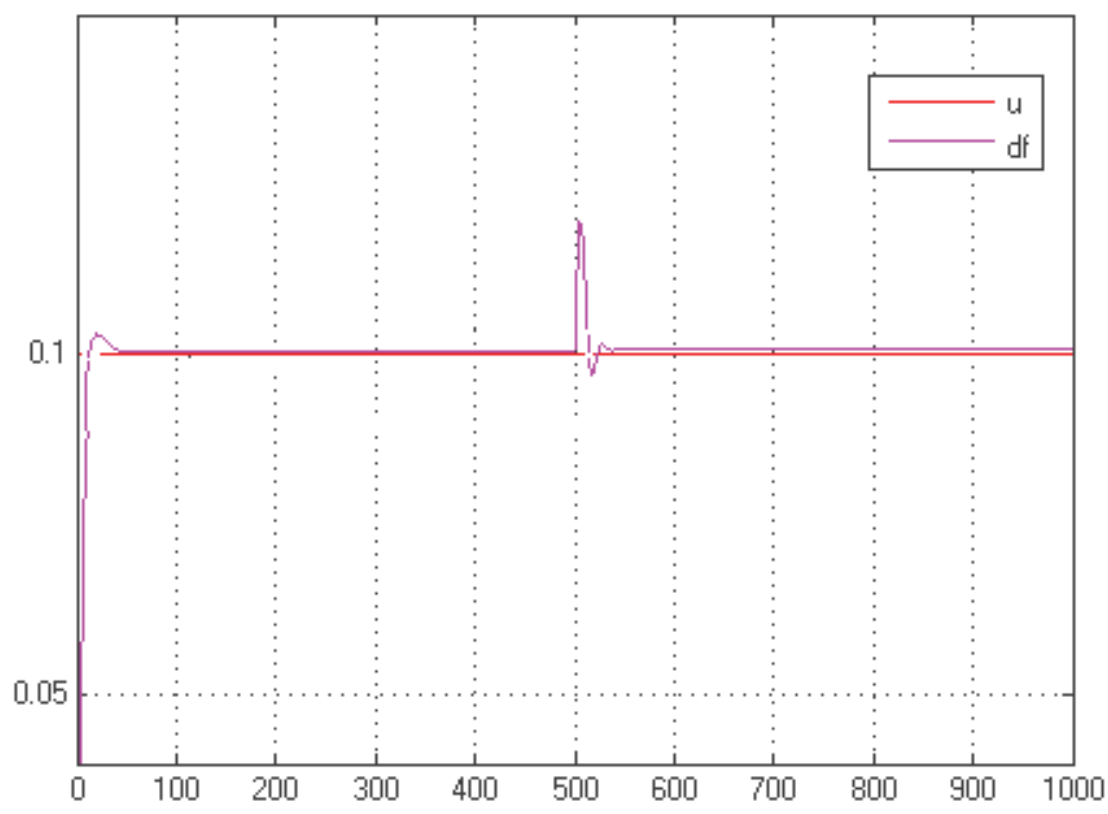

Figure 9. Reaction of system during change of loading

\section{Analysis of the Obtained Results}

During the conducted experiment, smooth work of the specification is observed. The time for establishment of system performs preliminarily set requirements (the transient process must damp within the framework of $25 \mathrm{~s}$. Because of its astatic character, the system exactly work of the specification - without overcontrol and shock. In the controlling signal, there is availability of oscillations (fluctuations), which is due to external actions (mostly noise from measurement, that is characteristic of the real system).

\section{Conclusion}

The synthesis and the realization of fuzzy PD controller for controlling of cross-flow water turbine have been presented in this paper. Control according to angular velocity of rotation of the turbine shaft has been applied. The concrete experiments have been carried out. It is shown that the designed PD controller is coped successfully with the set requirements. 


\section{References}

1. Harris, C. J., C. G. Moore and M. Brown. Intelligent Control Aspects of Fuzzy Logic and Neural Nets. London, World Scientific, 1994

2. Driankov, D., H. Hellendron and M. Reinfrank. An Introduction to Fuzzy Control. Heidelberg. Springer Verlag, 1993.

3. Dichev, D., H. Koev, T. Bakalova. A Kalman Filter-Based Algorithm for Measuring the Parameters of Moving Objects. - Measurement Science Review, 15, 2015, 1, 19-26.

4. Mamdani, E. H. Application of Fuzzy Algorithms for Control of Simple Dynamic Plant. - Proceedings IEEE, 121, 1974, No. 12. 5. George, K. I. Mann, Bao-Gang Hu and Raymond G. Gosine, Analysis of Direct Action Man, and Cybernetics - Part B: Cybernetics, 29, June 1999, No. 3.

6. Kishor, N., R. Saini and S. Singh. Optimal Pole Shift Control in Application to a Hydro Power Plant. - Journal of Electrical Engineering, 56, 2005, No. 11-12, 290-297.

7. Klimentov, K, G. Popov, K. Tujarov. Equations of Characteristics of Centrifugal Pumps. - Energetika, 2008, No. 6-7, 60-63.

8. Mansoor, S. Modelling of a Multiple Pump-Storage Units to a Power System. Conference Proceeding PREP'99, Manchester, England, 5-7 January 1999, 412-415.

9. Nielsen, T. Limitation of the Load Regulating Ability of Water Power Plants. $10^{\text {th }}$ International Meeting of the Work Group on the Behaviour of Hydraulic Machinery under Steady Oscilatory Conditions, Trondheim, Norway, 26-28 June 2001.

10. Nicolet, C., J. Prenat, F. Avellan, A. Sapin. A New Tool for the Simulation of Dynamic Behaviour of Hydroelectric Power Plants. $10^{\text {th }}$ International Meeting of the Work Group on the Behaviour of Hydraulic Machinery under Steady Oscilatory Conditions, Trondheim, Norway, 26-28 June 2001.

11. Ormandzhiev, K., P. Ivanov. Control Optimization of Parallel Working Water Turbines Fed by Means of Common Pressure Pipeline. HEFAT, Victoria Falls, 2003.

12. Ormandzhiev, K. Identification of the Parameters about Automatic Control System of Water Turbine. $9^{\text {th }}$ International Conference „Research and Development in Mechanical Industry“ RaDMI 2008, 14-17 September 2008, U•ice, Serbia, 2008.

13. Ormandzhiev, K. Dynamical Processes in Automatic System for Governing of Cross-flow Turbine. $11^{\text {th }}$ International Conference „Research and Development in Mechanical Industry“ RaDMI, 15-18 September, Sokobanja, Serbia, 2011.

14. Popov, G., K. Klimentov, B. Kostov. Methods to Estimate the Energy Consumption in Regulating the Flow Rate of Pump Systems. $10^{\text {th }}$ Anniversary International Conference an Accomplishment in Electrical and Mechanical Engineering and Information Technology, DEMI'2011. Banja Luka, May 2011, 495-500.

15. Yordanov, S, K. Ormandzhiev. Multiparameter the Control of the Cross-Flow Turbine. Conference ICEST 2005, Serbia and Montenegro, Niš, 28-30 June 2005, 139-142.

16. Weber, H., F. Ptillwitz, M. Hladky, H. Asal. Development of Reality Oriented Simulation Models of Power Plants and Power System for Restoration Studies. IFAC Symposium on Power Plants \& Power System Control, Brussels, Belgium, 26-29 April 2000.
Manuscript received on 13.06.2017

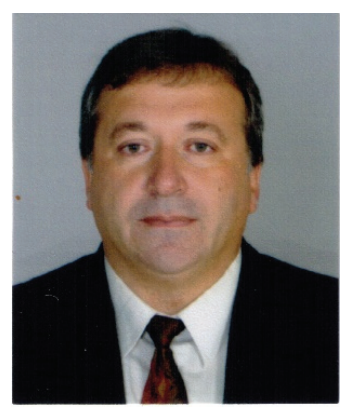

Assoc. Prof. Krasimir Ormandzhiev, PhD, Eng., has graduated from Technical University of Gabrovo, Faculty of Mechanical and Precision Engineering, speciality Hydraulic and Pneumatic Engineering. Since 1994 he is working at Technical University of Gabrovo in Department of Power Engineering. In 2010 he received PhD degree. His research interests are in the field of automatic hydraulic and pneumatic systems with classical and fuzzy control.

Contacts:

e-mail:khormandzhiev@abv.bg

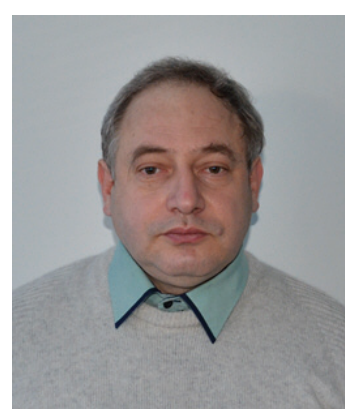

Assoc. Prof. Stanimir Yordanov, PhD, Eng., has graduated from Technical University of Gabrovo, Faculty of Electrical Engineering and Electronics, speciality Computing Technique. In 2005 he defended a doctor dissertation on "Computer system for quality control of the processing and monitoring of the processes in electrotechnological systems". Since 1993 he is working at Technical University of Gabrovo in Department of Automation, Information and Control Systems. His research interests are in the field of intelligent systems and processes control.

Contacts: e-mail: sjjordanov@mail.bg

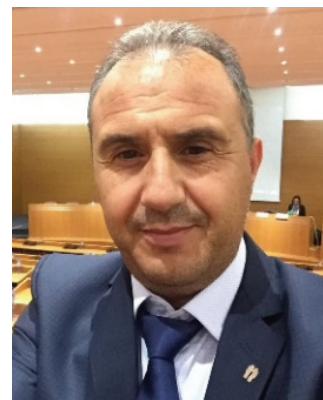

Stoian Stoianov was born in 1968 in Radnevo, Bulgaria. He has graduated from Technical University of Gabrovo, Faculty of Mechanical and Precision Engineering, speciality Hydraulic and Pneumatic Engineering. He is working at coal mining company Mini MaritsaIztok EAD. His research interests are in the field of automatic hydraulic and pneumatic systems.

Contacts: e-mail: stoianov_68@mail.bg 